\title{
Serious Adverse Events: What to Expect in Clinical Trials with Participation of Multiple Sclerosis Patients?
}

\author{
Daniella Bajzath ${ }^{1}$, Gyorgy Andor ${ }^{2}$ and Maxim Belotserkovskiy*3 \\ ${ }^{1}$ Department of Medical Affairs, Germany \\ ${ }^{2}$ Department of Finance and Accounting, Eotvos Lorand University, Hungary \\ ${ }^{3}$ Department of Medical Affairs Division, Germany
}

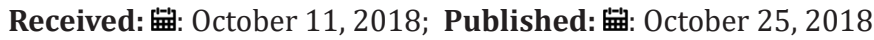

*Corresponding author: Maxim Belotserkovskiy, Department of Medical Affairs Division, Germany

\begin{abstract}
Multiple sclerosis is an inflammatory disease attacking the central nervous system, with a slightly reduced life-expectancy, affecting 2.1 million people worldwide, with female prevalence. A retrospective analysis summarizes safety data from 11 completed clinical trials with an aim to give an overview about Serious Adverse Event occurrence in study subjects, also detailing the events according to System Organ Class.

Abbreviations: SAE: Serious Adverse Event; MedDRA: Medical Dictionary for Regulatory Activities; SOC: System Organ Class; IMP: Investigational Medicinal Product; AEs: Adverse Events
\end{abstract}

\section{Introduction}

\section{Multiple Sclerosis}

Multiple sclerosis is an inflammatory disease attacking myelinated axons in the central nervous system, destroying the myelin and the axon in variable degrees. It causes significant physical disability within $20-25$ years in more than $30 \%$ of patients. The cause of multiple sclerosis is still unknown; it can be triggered or perpetuated by an as-yet-unidentified environmental factor in a genetically predisposed person. MS is considered an autoimmune disease; the immune system attacks the myelin sheets in the brain or spinal cord. Multiple sclerosis is most commonly diagnosed in people in their 20 s and 30 s. It is a life-time condition; average life-expectancy is slightly reduced. Worldwide, approximately 2.1 million people are affected, with prevalence in women [1].

\section{Clinical Trials}

According to the definition in ICH GCP, in clinical trials only those adverse events (AEs) are assessed as serious (SAEs) that occurred after any dose of the Investigational Medicinal Product (IMP) and resulted in death, were life-threatening, required inpatient hospitalization or prolongation of existing hospitalization, resulted in persistent or significant disability/incapacity or caused a congenital anomaly/birth defect. [2] Some of SAEs, which are assessed by investigators as related to the IMP, and also unexpected from regulatory standpoint - never registered before with the IMP
- were accounted separately as they require expedited reporting to Regulatory Authorities, Ethics Committees, and Investigators participating in the studies with this IMP [2]. Pregnancies, which are actually not SAEs but require expedited reporting by the investigators and close follow-up until delivery and perinatal period of the baby were also included into account. A great deal of publications discusses the safety findings of clinical trials, but they do not specify serious events in detail. [3-6] This retrospective analysis provides an overview on the number and type of serious AEs in clinical trials with general multiple sclerosis patient population and is independent on the study drug or treatment arm.

\section{Retrospective SAE Analysis}

Retrospective analysis of SAEs was performed from data of 11 completed Phase II and III clinical trials completed with multiple sclerosis patients within the period of 2008-2018. A total of 4463 subjects in the age of 18 and 55, from Europe, Australia, North America and South Africa were enrolled in the studies (Table 1).

During the analyzed trials 1361 SAEs were registered in $14.5 \%$ of treated subjects. One fourth of these SAEs were assessed as related to the investigational drug and unexpected (SARs), affecting almost $5 \%$ of the patients. A high amount of pregnancies was reported, 250 cases in $4.1 \%$ of all study subjects, in female subjects and female partners of male subjects. The most common 
seriousness criterion observed was hospitalization or prolongation of existing hospitalization. The most common disease groups according to System Organ Class (SOC) analysis using the Medical Dictionary for Regulatory Activities (MedDRA) were Infections and infestations and Nervous systems disorders. 10\% of the reported SAEs were due to different sorts of infections: Pneumonia, Herpes zoster, Sepsis, Urinary tract infection, Cellulitis, Gastroenteritis and Appendicitis.

Table 1: Statistical results.

\begin{tabular}{|c|c|}
\hline Category & Total \\
\hline Patients & 4463 \\
\hline SAEs & 1361 \\
\hline Number of Patients with SAEs & 645 \\
\hline SARs (drug-related SAEs) & 324 \\
\hline Number of Patients with SARs & 202 \\
\hline Deaths & 13 \\
\hline Pregnancies & 184 \\
\hline
\end{tabular}

\section{Note:}

$14.5 \%$ of all patients

$23.8 \%$ of all SAEs

$4.5 \%$ of all patients

$0.3 \% \quad$ of all patients

$4.1 \%$ of all patients

The high occurrence of any kind of infection in MS patients is likely due to the fact that the drugs used for treatment have the common side effect of reducing white blood cell count. $8 \%$ of all SAEs (excluding cases of MS relapse) were connected to various illnesses of the nervous system. The explanation to their high occurrence can be either because of the inefficacy of the study drug or randomization into the placebo treatment arm. 13 subjects were died while in the trial, representing $0.35 \%$ of all treated patients.
The main reasons for death were due to Infections and infestations in $25 \%$ of the death cases. The rest of the cases belonged to the following disease groups: Blood and lymphatic system disorders; Neoplasms benign, malignant and unspecified; Nervous system disorders; Psychiatric disorders and Respiratory, thoracic and mediastinal disorders.

\section{Conclusion}

This retrospective analysis of SAEs in clinical trials with multiple sclerosis, an inflammatory disease attacking the central nervous system, aimed to provide safety data from 11 clinical trials. SAEs were registered in $14.5 \%$ of treated patients, and quarter of them assessed as related to the study therapy. Most common SAE causes were Infections and infestations and Nervous systems disorders. $0.35 \%$ of treated patients died and the main reason was again Infections and infestations. The high prevalence of infections could be accounted for the side-effects of MS treatment.

\section{References}

1. Richard Nicholas, Waqae Rashid (2013) Multiple sclerosis. American Family Physician 87(10): 712-714

2. (2016) Guideline, ICH Harmonised. Guideline for good clinical practice E6 (R2).

3. C Confavreux, DK Li, MS Freedman, $\mathrm{P}$ Truffinet, $\mathrm{H}$ Benzerdjeb, et al. (2012) Long-term follow-up of a phase 2 study of oral teriflunomide in relapsing multiple sclerosis: safety and efficacy result up to 8.5 years. 18(9): 1278-1289.

4. R Gold, EW Radue, G Giovannoni, K Selmaj, E Havrdova, et al. (2016) Safety and efficacy of daclizumab in relapsing-remitting multiple sclerosis: 3-year results from the SELECTED open-label extension study. Sl BMC Neurology 16: 117.

5. J Salzer, R Svenningsson, P Alping, L Novakova, A Björck, et al. (2015) Rituximab in multiple sclerosis: A retrospective observational study on safety and efficacy. Neurology 87(20): 2074-2081.

6. T Olsson, A Boster, Ó Fernández, MS Freedman, C Pozzilli, et al. (2014) Oral ponesimod in relapsin-remitting multiple sclerosis: a randomized phase II trial. J Neurol Neurosurg Psychiatry 85(11): 1198-1208.
ISSN: 2574-1241

DOI: 10.26717/BJSTR.2018.10.001946

Maxim Belotserkovskiy. Biomed J Sci \& Tech Res

This work is licensed under Creative Commons Attribution 4.0 License

Submission Link: https://biomedres.us/submit-manuscript.php

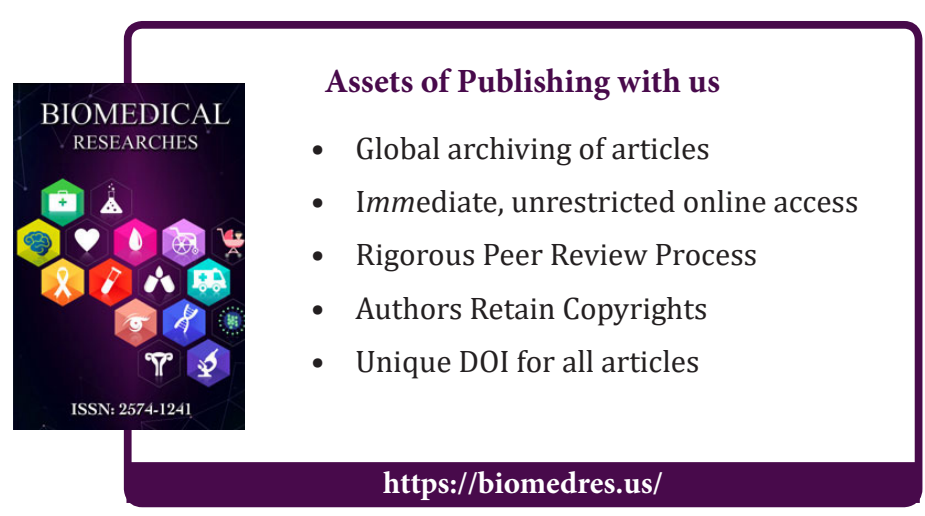

